\title{
SUMMATION BY PARTS, PROJECTIONS, AND STABILITY. II
}

\author{
PELLE OLSSON
}

\begin{abstract}
In this paper we prove strict stability of high-order finite difference approximations of parabolic and symmetric hyperbolic systems of partial differential equations on bounded, curvilinear domains in two space dimensions. The boundary need not be smooth. We also show how to derive strict stability estimates for inhomogeneous boundary conditions.
\end{abstract}

\section{StRICT STABILITY IN SEVERAL SPACE DIMENSIONS}

In [2] we proved stability for high-order finite difference approximations of hyperbolic and parabolic systems using certain projections and difference operators satisfying a summation-by-parts rule. In one space dimension we showed how to obtain strict stability. The aim of this paper is to prove strict stability in several space dimensions. Furthermore, it will also be demonstrated how to handle inhomogeneous boundary conditions. We limit ourselves to two space dimensions for convenience.

The purpose of strict stability is to ensure the same growth rate of the discrete and analytic solutions. If the analytic problem is defined on a curvilinear domain $\Omega$ with boundary $\Gamma$ (cf. Fig. 1 on next page), then there must exist a diffeomorphism $\xi=\xi(x)$ of $\Omega$ onto the unit square $(0,1) \times(0,1)$ in order for the finite difference method to be well defined. Consequently, a constantcoefficient problem in the original domain may be transformed to a variablecoefficient problem on the unit square, which may account for a nonphysical growth in the discrete estimate.

Let $\xi=\xi(x)$ be a diffeomorphism of $\Omega$ onto $I=(0,1) \times(0,1)$. The following identities are readily established:

$$
\begin{array}{ll}
\frac{\partial x_{1}}{\partial \xi_{1}}=J^{-1} \frac{\partial \xi_{2}}{\partial x_{2}}, & \frac{\partial x_{2}}{\partial \xi_{1}}=-J^{-1} \frac{\partial \xi_{2}}{\partial x_{1}}, \\
\frac{\partial x_{1}}{\partial \xi_{2}}=-J^{-1} \frac{\partial \xi_{1}}{\partial x_{2}}, & \frac{\partial x_{2}}{\partial \xi_{2}}=J^{-1} \frac{\partial \xi_{1}}{\partial x_{1}},
\end{array}
$$

which in turn implies

$$
\sum_{i=1}^{2}\left(J^{-1} \partial \xi_{i}\right)_{\xi_{i}}=0
$$

Received by the editor January 19, 1994 and, in revised form, August 5, 1994.

1991 Mathematics Subject Classification. Primary 65M06, 65M12.

This work has been sponsored by NASA under contract No. NAS 2-13721. 


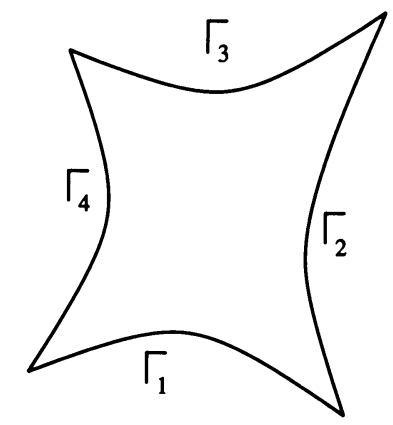

FIGURE 1. Domain $\Omega$ with boundary $\Gamma=\bigcup_{i=1}^{4} \Gamma_{i}$

where $\partial$ denotes the two-dimensional gradient operator. We require that $\xi(x)$ be uniformly nonsingular, i. e., there exists a constant $\delta>0$ such that $J^{-1} \geq \delta$ on $\Omega$. For later use we record the normal and tangential derivatives $u_{n_{i}}$ and $u_{\tau_{i}}$ at the boundaries corresponding to $\xi_{i}=0, i=1,2$ :

$$
\begin{aligned}
& u_{\tau_{j}}=(-1)^{i} u_{\xi_{j}} /\left|x_{\xi_{j}}\right|, \\
& u_{n_{i}}=-\left(\partial \xi_{i} \cdot \partial \xi_{i} u_{\xi_{i}}+\partial \xi_{i} \cdot \partial \xi_{j} u_{\xi_{j}}\right) /\left|\partial \xi_{i}\right|,
\end{aligned} \quad i, j=1,2, \quad j \neq i,
$$

where the boundary $\Gamma$ of the domain $\Omega$ has been parameterized in the positive direction.

The analytic scalar product obeys

$$
(u, v)=\int_{\Omega} u^{T}(x) v(x) d x=\int_{I} u^{T}(x(\xi)) v(x(\xi)) J^{-1} d \xi,
$$

which suggests the following semidiscrete scalar product:

$$
\langle u, v\rangle_{h} \equiv\left(u, J^{-1} v\right)_{h}=\left(J^{-1} u, v\right)_{h},
$$

where

$$
(u, v)_{h}=h_{1} h_{2} \sum_{i, j=0}^{\nu} \sigma_{i} \sigma_{j} u_{i j}^{T} v_{i j} \equiv h u^{T} \Sigma v, \quad h_{1}=\Delta \xi_{1}, \quad h_{2}=\Delta \xi_{2} .
$$

We have assumed the same number of grid points in both coordinate directions for convenience. The coefficients $\sigma_{j}$ have been determined such that the difference operators $D_{i}$ approximating $\partial / \partial \xi_{i}, i=1,2$, satisfy a summation-byparts rule. Notational conventions for $D$ and the definition of the summationby-parts rule can be found in [2]; $u^{T}=\left(\begin{array}{llll}u_{0}^{T} & \ldots & u_{\nu}^{T}\end{array}\right), u_{j}^{T}=\left(\begin{array}{lll}u_{0 j}^{T} & \ldots & u_{\nu j}^{T}\end{array}\right)$, $u_{i j} \in \mathbf{R}^{d}$; the matrix representation of $J^{-1}$ is

$$
J^{-1}=\left(\begin{array}{ccc}
J_{0}^{-1} & & \\
& \ddots & \\
& & J_{\nu}^{-1}
\end{array}\right), \quad J_{j}^{-1}=\left(\begin{array}{ccc}
J_{0 j}^{-1} I & & \\
& \ddots & \\
& & J_{\nu j}^{-1} I
\end{array}\right), \quad I \in \mathbf{R}^{d},
$$

where

$$
J_{i j}^{-1}=J^{-1}\left(i h_{1}, j h_{2}\right)=\operatorname{det}\left(\frac{\partial x}{\partial \xi}\left(i h_{1}, j h_{2}\right)\right)
$$


Similarly,

$$
\Sigma=\left(\begin{array}{ccc}
\sigma_{0} \Sigma_{0} & & \\
& \ddots & \\
& & \sigma_{\nu} \Sigma_{\nu}
\end{array}\right), \quad \Sigma_{j}=\left(\begin{array}{ccc}
\sigma_{0} I & & \\
& \ddots & \\
& & \sigma_{\nu} I
\end{array}\right), \quad j=0, \ldots, \nu .
$$

Thus, each grid point in (1.4) is scaled with the cell volume.

The analytic boundary integrals can be parameterized as

$$
\int_{\Gamma_{1}} u^{T}(x) v(x) d s=\int_{0}^{1} u^{T}\left(x\left(\xi_{1}, 0\right)\right) v\left(x\left(\xi_{1}, 0\right)\right)\left|x_{\xi_{1}}\left(\xi_{1}, 0\right)\right| d \xi_{1} .
$$

Hence, it is natural to define the boundary scalar product as

$$
\langle u, v\rangle_{\Gamma} \equiv \sum_{j=0}^{\nu} \sigma_{j}\left(s_{0 j} u_{0 j}^{T} v_{0 j}+s_{\nu j} u_{\nu j}^{T} v_{\nu j}\right)+\sum_{i=0}^{\nu} \sigma_{i}\left(s_{i 0} u_{i 0}^{T} v_{i 0}+s_{i \nu} u_{i \nu}^{T} v_{i \nu}\right),
$$

where the arc lengths are defined as

$$
s_{0 j}=\left|x_{\xi_{2}}\left(0, j h_{2}\right)\right| h_{2}, \quad s_{i 0}=\left|x_{\xi_{1}}\left(i h_{1}, 0\right)\right| h_{1},
$$

with similar definitions for $s_{\nu j}$ and $s_{i \nu}$.

It was shown in $[2, \S 2.2]$ that the projection $P$ representing the analytic boundary conditions can be written as

$$
P=I-\Sigma^{-1} L\left(L^{T} \Sigma^{-1} L\right)^{-1} L^{T},
$$

where $L$ is the discretization of the analytic boundary operator. It follows that $(u, P v)_{h}=(P u, v)_{h}$. It suffices to consider solutions that are supported only in a neighborhood of $\left(x_{1}(0,0), x_{2}(0,0)\right) ; P$ is assumed to be independent of $t$. In two space dimensions the general form of $L$ is

$$
L=\left(\begin{array}{llllll}
L_{10} & \ldots & L_{1 r} & L_{20} & \ldots & L_{2 r}
\end{array}\right) \in \mathrm{R}^{(\nu+1)^{2} d \times 2(r+1) d},
$$

where $L_{1 j}$ and $L_{2 i}$ denote the boundary operators at $x\left(0, j h_{2}\right)$ and $x\left(i h_{1}, 0\right)$; $r$ is a constant depending on the approximation order of the boundary conditions (typically the number of grid points along a coordinate line involved in the approximation of the normal derivative). However, $r$ does not depend on the mesh sizes $h_{1}, h_{2}$. Furthermore,

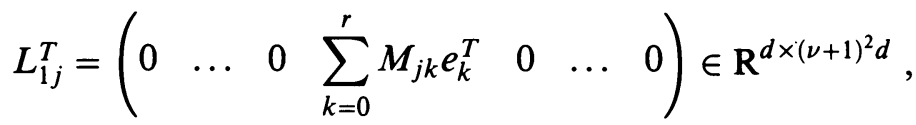

$$
\begin{aligned}
& L_{2 i}^{T}=\left(\begin{array}{llllll}
N_{i 0} e_{i}^{T} & \ldots & N_{i r} e_{i}^{T} & 0 & \ldots & 0
\end{array}\right) \in \mathrm{R}^{d \times(\nu+1)^{2} d} .
\end{aligned}
$$

Here, $e_{i}^{T}=\left(\begin{array}{lllllll}0 & \ldots & 0 & I & 0 & \ldots & 0\end{array}\right) \in \mathrm{R}^{d \times(\nu+1) d}, M_{j k}, N_{i k} \in \mathrm{R}^{d \times d}$.

In order to prove stability, we must have $P v=v$. Since $v$ will be the solution of equations like (1.13), it follows that $P J^{-1} v=J^{-1} v$. Therefore, it is natural to require

$$
P J^{-1}=J^{-1} P
$$


For a general $P$ this identity expresses a compatibility condition between the analytic boundary conditions and the mapping $\xi(x)$. Let $P$ be given by (1.8) and (1.9). Then (1.10) certainly holds if

$$
\begin{array}{ll}
J_{i j} \equiv J\left(x\left(i h_{1}, j h_{2}\right)\right)=J_{i 0}, & i=0, \ldots, \nu, \quad j=0, \ldots, r, \\
J_{i j} \equiv J\left(x\left(i h_{1}, j h_{2}\right)\right)=J_{0 j}, & i=0, \ldots, r, \quad j=0, \ldots, \nu,
\end{array}
$$

which states that the mapping $\xi(x)$ is locally isochoric, i. e., volume preserving, in the $x_{\xi_{i}}$-direction at the boundary, where $\partial / \partial \xi_{i}$ is the nontangential derivative. In case of characteristic boundary conditions and Dirichlet conditions we have $r=0$, and (1.11) is trivially satisfied. For general boundary conditions, however, (1.11) couples the boundary operator to the grid transformation.

1.1. Symmetric hyperbolic systems. Using (1.2), we recast

$$
u_{t}=\sum_{i=1}^{2} A_{i} u_{x_{i}}+F, \quad x \in \Omega, \quad A_{i}^{T}=A_{i},
$$

into a form that eliminates the need for the commutator in the semidiscrete case

$$
\left(J^{-1} u\right)_{t}=\frac{1}{2} \sum_{i=1}^{2}\left(\left(J^{-1} B_{i} u\right)_{\xi_{i}}+J^{-1} B_{i} u_{\xi_{i}}\right)-\frac{1}{2} J^{-1} \operatorname{div}(A) u+J^{-1} F,
$$

where

$$
B_{i}=\partial \xi_{i} \cdot A=\frac{\partial \xi_{i}}{\partial x_{1}} A_{1}+\frac{\partial \xi_{i}}{\partial x_{2}} A_{2}, \quad \operatorname{div}(A)=\partial \cdot A=\frac{\partial A_{1}}{\partial x_{1}}+\frac{\partial A_{2}}{\partial x_{2}} .
$$

Define the characteristic variables as $\varphi(x, t)=Q^{T}(x) u(x, t), x \in \Gamma$, where $Q$ are the orthogonal eigenvectors (assumed to be time-independent) of $B \equiv$ $n_{1} A_{1}+n_{2} A_{2} ; n$ is the outward unit normal at $\Gamma$. At each boundary point $x_{i j}$ it is assumed that the eigenvalues of $B\left(x_{i j}\right)$ satisfy $\left|\lambda\left(x_{i j}\right)\right| \geq \gamma_{i j}$; the significance of this assumption is explained in $[2, \S \S 3.1,4.1]$. The characteristic boundary conditions can then be expressed as

$$
\varphi_{I}(x, t)=S(x) \varphi_{I I}(x, t),
$$

where $S(x)$ is assumed to be "small"; $\varphi_{I}(x, t), \varphi_{I I}(x, t)$ denote the in- and outgoing characteristic variables. In the original variables we thus have

$$
L(x) u(x, t) \equiv\left(Q_{I}^{T}(x)-S(x) Q_{I I}^{T}(x)\right) u(x, t)=0, \quad x \in \Gamma .
$$

The smallness assumption on $S(x)$ implies that $L(x)$ has full rank. At the corner $x_{00} \equiv x(0,0)$ we require that the characteristic boundary conditions be satisfied for $\varphi\left(x_{00}, t\right)=Q_{i}^{T}\left(x_{00}\right) u\left(x_{00}, t\right), i=1,2$, where $Q_{i}$ are the eigenvectors of $B\left(x_{00}\right), n=n^{(i)}=-\partial \xi_{i} /\left|\partial \xi_{i}\right|$ being the two normals at the 
corner. This means that two sets of boundary conditions

$$
L_{1} u \equiv\left(Q_{1, I}^{T}-S Q_{1, I}^{T}\right) u=0, \quad L_{2} u \equiv\left(Q_{2, I}^{T}-S Q_{2, I I}^{T}\right) u=0,
$$

are prescribed. At the corner the resulting boundary operator may thus be written as

$$
L\left(x_{00}\right)=\left(\begin{array}{l}
L_{1}\left(x_{00}\right) \\
L_{2}\left(x_{00}\right)
\end{array}\right)
$$

It will be assumed that $L\left(x_{00}\right)$ has full rank, which occurs for the Euler equations at solid corners, where it is natural to require that both normal components of the velocity field be zero. This assumption simplifies the computations that follow. Furthermore, a rank-deficient operator $L\left(x_{00}\right)$ implies that there are redundant boundary conditions embedded in $L_{1}\left(x_{00}\right)$ and $L_{2}\left(x_{00}\right)$. In that case the redundancies should be eliminated before forming $L\left(x_{00}\right)$ from $L_{1}\left(x_{00}\right)$ and $L_{2}\left(x_{00}\right)$.

For the semidiscrete case the boundary operator is defined by

$$
\begin{aligned}
\left(L^{T} v\right)_{i j}=L\left(x_{i j}\right) v_{i j}, \quad i & =0, \nu, \quad j=1, \ldots, \nu-1, \text { and } \\
j & =0, \nu, \quad i=0, \ldots, \nu,
\end{aligned}
$$

where $L$ now has full rank. For details on the explicit structure of $L$ we refer to $[2, \S 4.1]$. The projection operator $P$ is then defined by (1.8).

Equation (1.12) is discretized as

$$
\left(J^{-1} v\right)_{t}=P\left(\frac{1}{2} \sum_{i=1}^{2}\left(D_{i} J^{-1} B_{i} v+J^{-1} B_{i} D_{i} v\right)-\frac{1}{2} J^{-1} C v+J^{-1} F\right)
$$

with

$$
C=\left(\begin{array}{lll}
\operatorname{div}(A)(x(0,0), t) & & \\
& \ddots & \\
& & \operatorname{div}(A)(x(1,1), t)
\end{array}\right)
$$

the $B_{i}$ 's are defined analogously.

Proposition 1.1. The approximation (1.13) is strictly stable.

Proof. The energy method yields (using $P J^{-1} v=J^{-1} v, P J^{-1}=J^{-1} P \Longrightarrow$ $P v=v)$

$$
\begin{aligned}
\frac{d}{d t}\langle v, v\rangle_{h}=\sum_{i=1}^{2} & \left(\left(v, D_{i} J^{-1} B_{i} v\right)_{h}+\left(v, J^{-1} B_{i} D_{i} v\right)_{h}\right) \\
& -\left(v, J^{-1} C v\right)_{h}+2\left(v, J^{-1} F\right)_{h} .
\end{aligned}
$$

But (using $B_{1}\left(0, j h_{2}\right)$ instead of $B_{1}\left(x\left(0, j h_{2}\right), t\right)$ and so forth to make the notation less cumbersome)

$$
\left(v, D_{1} J^{-1} B_{1} v\right)_{h}=-h_{2} \sum_{j=0}^{\nu} \sigma_{j} v_{0 j}^{T} J^{-1}\left(0, j h_{2}\right) B_{1}\left(0, j h_{2}\right) v_{0 j}-\left(D_{1} v, J^{-1} B_{1} v\right)_{h}
$$


Since diagonal scalar products are used, it follows that $\left(B_{1}^{T}=B_{1}\right)$

$$
\left(D_{1} v, J^{-1} B_{1} v\right)_{h}=\left(B_{1} J^{-1} D_{1} v, v\right)_{h}=\left(J^{-1} B_{1} D_{1} v, v\right)_{h},
$$

where the last equality follows since $B_{1}$ and $J^{-1}$ commute. Thus,

$$
\left(v, D_{1} J^{-1} B_{1} v\right)_{h}=-h_{2} \sum_{j=0}^{\nu} \sigma_{j} v_{0 j}^{T} J^{-1}\left(0, j h_{2}\right) B_{1}\left(0, j h_{2}\right) v_{0 j}-\left(J^{-1} B_{1} D_{1} v, v\right)_{h}
$$

with a similar relation for $\left(v, D_{2} J^{-1} B_{2} v\right)_{h}$. We thus arrive at

$$
\begin{aligned}
\frac{d}{d t}\langle v, v\rangle_{h} \leq & -h_{2} \sum_{j=0}^{\nu} \sigma_{j} v_{0 j}^{T} J^{-1}\left(0, j h_{2}\right) B_{1}\left(0, j h_{2}\right) v_{0 j} \\
& -h_{1} \sum_{i=0}^{\nu} \sigma_{i} v_{i 0}^{T} J^{-1}\left(i h_{1}, 0\right) B_{2}\left(i h_{1}, 0\right) v_{i 0} \\
& +\left(|\operatorname{div}(A)|_{\infty}+1\right)\langle v, v\rangle_{h}+\langle F, F\rangle_{h} .
\end{aligned}
$$

By means of (1.1) it follows that

$$
J^{-1} B_{1}=\frac{\partial x_{2}}{\partial \xi_{2}} A_{1}-\frac{\partial x_{1}}{\partial \xi_{2}} A_{2}
$$

Evidently, $x_{\xi_{2}}$ is a tangent vector of the curve $x\left(0, \xi_{2}\right)$. Hence,

$$
\left(\begin{array}{ll}
-\frac{\partial x_{2}}{\partial \xi_{2}} & \frac{\partial x_{1}}{\partial \xi_{2}}
\end{array}\right)
$$

is an outward normal to $x\left(0, \xi_{2}\right) \in \Gamma$. The unit normal is then defined as

$$
\left(\begin{array}{ll}
n_{1} & n_{2}
\end{array}\right)=\left(\begin{array}{ll}
-\frac{\partial x_{2}}{\partial \xi_{2}} & \frac{\partial x_{1}}{\partial \xi_{2}}
\end{array}\right) /\left|x_{\xi_{2}}\right| .
$$

Using the definition of the arc length, we then obtain

$$
-h_{2} \sum_{j=0}^{\nu} \sigma_{j} v_{0 j}^{T} J^{-1}\left(0, j h_{2}\right) B_{1}\left(0, j h_{2}\right) v_{0 j}=\sum_{j=0}^{\nu} \sigma_{j} s_{0 j} v_{0 j}^{T}\left(n_{1} A_{1}+n_{2} A_{2}\right) v_{0 j} .
$$

But $P v=v$ implies that the analytic boundary conditions are satisfied, whence

$$
v_{0 j}^{T}\left(n_{1} A_{1}+n_{2} A_{2}\right) v_{0 j} \leq-\gamma_{j}\left|v_{0 j}\right|^{2} .
$$

Letting $\gamma \equiv \inf \left(\gamma_{j}\right)>0$ implies

$$
-h_{2} \sum_{j=0}^{\nu} \sigma_{j} v_{0 j}^{T} J^{-1}\left(0, j h_{2}\right) B_{1}\left(0, j h_{2}\right) v_{0 j} \leq-\gamma \sum_{j=0}^{\nu} \sigma_{j} s_{0 j}\left|v_{0 j}\right|^{2} .
$$

We have thus established

$$
\frac{d}{d t}\langle v, v\rangle_{h}+\gamma\langle v, v\rangle_{\Gamma} \leq\left(|\operatorname{div}(A)|_{\infty}+1\right)\langle v, v\rangle_{h}+\langle F, F\rangle_{h} .
$$

This is exactly the same estimate that one would get in the analytic case, and the proposition has been proved. 
1.2. The heat equation. In curvilinear coordinates the heat equation $u_{t}=u_{x_{1} x_{1}}$ $+u_{x_{2} x_{2}}, x \in \Omega$, can be expressed as

$$
\left(J^{-1} u\right)_{t}=\sum_{i, k=1}^{2}\left(J^{-1} \frac{\partial \xi_{k}}{\partial x_{i}}\left(\sum_{j=1}^{2} \frac{\partial \xi_{j}}{\partial x_{i}} u_{\xi_{j}}\right)\right)_{\xi_{k}} .
$$

At the boundary the normal derivative is set to zero, i. e., $u_{n}(x, t)=0, x \in \Gamma$. Define

$$
M_{i j}=\left(\begin{array}{lll}
\frac{\partial \xi_{j}}{\partial x_{i}}(x(0,0)) & & \\
& \ddots & \\
& & \frac{\partial \xi_{j}}{\partial x_{i}}(x(1,1))
\end{array}\right)
$$

and

$$
\tilde{D}_{i}=\sum_{j=1}^{2} M_{i j} D_{j}
$$

Clearly, $\tilde{D}_{i}$ is a consistent approximation of $\partial / \partial x_{i}$. Define $L_{1 j}^{T} v \equiv\left(D_{1} v\right)_{0 j}$, $j=0, \ldots, r, L_{2 i}^{T} v \equiv\left(D_{2} v\right)_{i 0}, i=0, \ldots, r$, i. e.,

$$
\begin{aligned}
& L_{1 j}^{T}=\left(\begin{array}{lllllll}
0 & \ldots & 0 & \frac{1}{h_{1}} \sum_{k=0}^{r} d_{0 k} e_{k}^{T} & 0 & \ldots & 0
\end{array}\right), \quad \in \mathrm{R}^{1 \times(\nu+1)^{2}}, j=0, \ldots, r, \\
& L_{2 i}^{T}=\left(\begin{array}{llllll}
\frac{d_{00}}{h_{2}} e_{i}^{T} & \ldots & \frac{d_{0 r}}{h_{2}} e_{i}^{T} & 0 & \ldots & 0
\end{array}\right), \quad \in \mathrm{R}^{1 \times(\nu+1)^{2}}, i=0, \ldots, r,
\end{aligned}
$$

where $\left\{e_{i}\right\}$ is the canonical basis in $\mathrm{R}^{\nu+1}$. Then

$$
\begin{aligned}
& L_{n_{1} 0}=-\left|\partial \xi_{1}\right|_{00} L_{10}-\frac{\left(\partial \xi_{1} \cdot \partial \xi_{2}\right)_{00}}{\left|\partial \xi_{1}\right|_{00}} L_{20}, \\
& L_{n_{2} 0}=-\left|\partial \xi_{2}\right|_{00} L_{20}-\frac{\left(\partial \xi_{1} \cdot \partial \xi_{2}\right)_{00}}{\left|\partial \xi_{2}\right|_{00}} L_{10}
\end{aligned}
$$

are consistent approximations of the outward normals at the origin. At the remaining grid points of $\Gamma_{4}$ and $\Gamma_{1}$ we set

$$
L_{n_{1} j}=-\left|\partial \xi_{1}\right|_{0 j} L_{1 j}, \quad j=1, \ldots, r, \quad L_{n_{2} i}=-\left|\partial \xi_{2}\right|_{i 0} L_{2 i}, \quad i=1, \ldots, r .
$$

We note that for orthogonal grids these operators are discrete outward normal difference operators.

The boundary projection $P$ is defined by (1.8), where

$$
L=\left(\begin{array}{llllll}
L_{n_{1} 0} & \ldots & L_{n_{1} r} & L_{n_{2} 1} & \ldots & L_{n_{2} r}
\end{array}\right) .
$$

It should be noted that $P$ may no longer be unconditionally well defined since $L$ may be rank-deficient. Elementary computations show that

$$
\sum_{j=0}^{r} \alpha_{j} L_{n_{1} j}+\sum_{i=1}^{r} \beta_{i} L_{n_{2} i}=0
$$


implies

$$
\left(\left|\partial \xi_{1}\right|_{00}^{2} h_{2}+\left(\partial \xi_{1} \cdot \partial \xi_{2}\right)_{00} h_{1}\right) \alpha_{0}=0 .
$$

If $\left(\partial \xi_{1} \cdot \partial \xi_{2}\right)_{00}<0$, i. e., at acute corners, there is a possibility of a nonzero $\alpha_{0}$ if

$$
h_{2}=-\frac{\partial \xi_{1} \cdot \partial \xi_{2}}{\left|\partial \xi_{1}\right|^{2}} h_{1}
$$

Hence, at acute corners we assume $h_{1}$ and $h_{2}$ to be such that (1.16) does not hold. Furthermore,

$$
L=\left(\begin{array}{llllllll}
L_{n_{1} 0} & L_{n_{1} 1} & \ldots & L_{n_{1} r} & L_{n_{2} 0} & L_{n_{2} 1} & \ldots & L_{n_{2} r}
\end{array}\right)
$$

is rank-deficient. This is obvious if $\partial \xi_{1} \cdot \partial \xi_{2}= \pm\left|\partial \xi_{1}\right|\left|\partial \xi_{2}\right|$, because then $L_{n_{1} 0}= \pm L_{n_{2} 0}$. Otherwise, we note that

$$
\sum_{j=0}^{r} \alpha_{j} L_{n_{1} j}+\sum_{i=0}^{r} \beta_{i} L_{n_{2} i}=0
$$

is equivalent to

$$
\sum_{j=0}^{r} \alpha_{j}^{\prime} L_{1 j}+\sum_{i=1}^{r} \beta_{i}^{\prime} L_{2 i}=0,
$$

where the latter equation has the nontrivial solution $\alpha_{j}^{\prime}=d_{0 j}, \beta_{i}^{\prime}=-\left(h_{2} / h_{1}\right) d_{0 i}$ [2, Lemma 4. 1]. Here,

$$
\alpha_{j}^{\prime}=\left|\partial \xi_{1}\right|_{0 j} \alpha_{j}, \quad j=1, \ldots, r, \quad \beta_{i}^{\prime}=\left|\partial \xi_{2}\right|_{i 0} \beta_{i}, \quad i=1, \ldots, r,
$$

and

$$
\left(\begin{array}{cc}
\left|\partial \xi_{1}\right|_{00} & \frac{\left(\partial \xi_{1} \cdot \partial \xi_{2}\right)_{00}}{\left|\partial \xi_{2}\right|_{00}} \\
\frac{\left(\partial \xi_{1} \cdot \partial \xi_{2}\right)_{00}}{\left|\partial \xi_{1}\right|_{00}} & \left|\partial \xi_{2}\right|_{00}
\end{array}\right)\left(\begin{array}{l}
\alpha_{0} \\
\beta_{0}
\end{array}\right)=\left(\begin{array}{c}
\alpha_{0}^{\prime} \\
\beta_{0}^{\prime}
\end{array}\right),
$$

which has a unique solution if and only if $\left|\partial \xi_{1} \cdot \partial \xi_{2}\right|_{00}<\left|\partial \xi_{1}\right|_{00}\left|\partial \xi_{2}\right|_{00}$. This shows that

$$
L_{n_{2} 0} \in \operatorname{span}\left[\begin{array}{llllll}
L_{n_{1} 0} & \ldots & L_{n_{1} r} & L_{n_{2} 1} & \ldots & L_{n_{2} r}
\end{array}\right] \equiv \operatorname{span}[L] .
$$

The semidiscrete heat equation is now defined as

$$
\left(J^{-1} v\right)_{t}=P \sum_{i, k=1}^{2} D_{k}\left(J^{-1} M_{i k}\left(\sum_{j=1}^{2} M_{i j} D_{j} v\right)\right) .
$$

Proposition 1.2. Assume that the mapping $\xi(\Omega)=I$ is locally isochoric at the boundary in the sense of (1.11), and that the grid is orthogonal at the boundaries except at the corners. Then (1.17) is strictly stable. 
Proof. Since the transformation is locally isochoric at the boundary, we get $P v=v$. Thus, the energy method implies

$$
\frac{d}{d t}\langle v, v\rangle_{h}=2 \sum_{i, k=1}^{2}\left(v, D_{k} J^{-1} M_{i k} \tilde{D}_{i} v\right)_{h} .
$$

Summation by parts yields ( $v$ is assumed to have compact support)

$$
\begin{aligned}
\frac{d}{d t}\langle v, v\rangle_{h}= & -2 \sum_{i=1}^{2} h_{2} \sum_{l=0}^{\nu} \sigma_{l} v_{0 l}\left(J^{-1} M_{i 1} \tilde{D}_{i} v\right)_{0 l} \\
& -2 \sum_{i=1}^{2} h_{1} \sum_{l=0}^{\nu} \sigma_{l} v_{l 0}\left(J^{-1} M_{i 2} \tilde{D}_{i} v\right)_{l 0}-2 \sum_{i, k=1}^{2}\left(M_{i k} D_{k} v, J^{-1} \tilde{D}_{i} v\right)_{h} .
\end{aligned}
$$

Obviously,

$$
\sum_{i, k=1}^{2}\left(M_{i k} D_{k} v, J^{-1} \tilde{D}_{i} v\right)_{h}=\sum_{i=1}^{2}\left\langle\tilde{D}_{i} v, \tilde{D}_{i} v\right\rangle_{h} .
$$

Next we turn our attention to the boundary terms. We have

$$
\sum_{i=1}^{2}\left(J^{-1} M_{i 1} \tilde{D}_{i} v\right)_{0 l}=J_{0 l}^{-1}\left|\partial \xi_{1}\right|_{0 l}\left(\left|\partial \xi_{1}\right|_{0 l}\left(D_{1} v\right)_{0 l}+\frac{\left(\partial \xi_{1} \cdot \partial \xi_{2}\right)_{0 l}}{\left|\partial \xi_{1}\right|_{0 l}}\left(D_{2} v\right)_{0 l}\right) .
$$

The parenthetical expression is recognized as a discretization of the normal derivative (cf. (1.3)). The other boundary is treated analogously. At $\xi_{1}=0$ we thus define a "normal difference" operator $\tilde{D}_{n_{1}}$ through

$$
\left(\tilde{D}_{n_{1}} v\right)_{0 l}=-\left(\left|\partial \xi_{1}\right|_{0 l}\left(D_{1} v\right)_{0 l}+\frac{\left(\partial \xi_{1} \cdot \partial \xi_{2}\right)_{0 l}}{\left|\partial \xi_{1}\right|_{0 l}}\left(D_{2} v\right)_{0 l}\right)
$$

with a similar definition of $\tilde{D}_{n_{2}}$ at $\xi_{2}=0$. From (1.1) it follows that $J_{0 l}^{-1}\left|\partial \xi_{1}\right|=$ $\left|x_{\xi_{2}}\right|$. Hence,

$$
\frac{d}{d t}\langle v, v\rangle_{h}=2\left\langle v, \tilde{D}_{n} v\right\rangle_{\Gamma}-2 \sum_{i=1}^{2}\left\langle\tilde{D}_{i} v, \tilde{D}_{i} v\right\rangle_{h} .
$$

Using $v=P v$ and the orthogonality assumptions, we conclude that

$$
\begin{aligned}
& \left(\tilde{D}_{n_{1}} v\right)_{0 l}=-\left|\partial \xi_{1}\right|_{0 l} L_{1 l}^{T} v=L_{n_{l} l}^{T} v=0, \quad l>0 . \\
& \left(\tilde{D}_{n_{2}} v\right)_{l 0}=-\left|\partial \xi_{2}\right|_{l 0} L_{2 l}^{T} v=L_{n_{2} l}^{T} v=0,
\end{aligned}
$$

At the origin we have

$$
\begin{aligned}
& \left(\tilde{D}_{n_{1}} v\right)_{00}=L_{n_{1} 0}^{T} v=0, \\
& \left(\tilde{D}_{n_{2}} v\right)_{00}=L_{n_{2} 0}^{T} v=0,
\end{aligned}
$$

where $L_{n_{1} 0}^{T} v$ vanishes because of the construction of $P ; L_{n_{2} 0}^{T} v$ disappears since we have shown that $L_{n_{2} 0}$ belongs to the column space of $L$. Hence the boundary sum is identically zero, which proves the proposition.

Remark. It would still be possible to prove strict stability, even if the grid were not orthogonal at the boundary. To compensate for the loss of orthogonality, it 
is necessary to require that the grid be globally isochoric in a neighborhood of the boundary $\Gamma$.

1.3. General parabolic systems. When considering parabolic equations in general, tangential derivatives may appear in the boundary integrals, potentially calling for integration by parts once more. The occurrence of tangential derivatives depends on the coefficients of the original equation, the geometry, and the presence of mixed derivatives. These criteria are not independent of one another. The following simple example will illustrate this interdependence. Consider the parabolic model equation

$$
u_{t}=u_{x_{1} x_{1}}+u_{x_{1} x_{2}}+u_{x_{2} x_{2}}, \quad x \in \Omega,
$$

where $\Omega$ is diffeomorphic to the unit square; the boundary conditions are yet to be specified. The energy method gives (the cross term is integrated with respect to $x_{1}$ )

$$
\frac{d}{d t}\|u\|^{2}=2 \int_{\Gamma}\left(u u_{n}+n_{1} u u_{x_{2}}\right) d s-2 \int_{\Omega}\left(u_{x_{1}} u_{x_{1}}+u_{x_{1}} u_{x_{2}}+u_{x_{2}} u_{x_{2}}\right) d x .
$$

The normal and tangential derivatives are defined as

$$
\begin{aligned}
& \frac{\partial}{\partial n}=n_{1} \frac{\partial}{\partial x_{1}}+n_{2} \frac{\partial}{\partial x_{2}}, \quad \frac{\partial}{\partial x_{1}}=n_{1} \frac{\partial}{\partial n}+\tau_{1} \frac{\partial}{\partial \tau}, \\
& \frac{\partial}{\partial \tau}=\tau_{1} \frac{\partial}{\partial x_{1}}+\tau_{2} \frac{\partial}{\partial x_{2}}, \Longleftrightarrow \frac{\partial}{\partial x_{2}}=n_{2} \frac{\partial}{\partial n}+\tau_{2} \frac{\partial}{\partial \tau},
\end{aligned}
$$

where $n$ is the outward unit normal as usual; the unit tangential $\tau$ is chosen corresponding to a positive orientation of $\Gamma$. Thus,

$$
\begin{aligned}
& \tau_{1}=-n_{2}, \\
& \tau_{2}=n_{1} .
\end{aligned}
$$

If, on the other hand, the cross term is integrated with respect to $x_{2}$, we obtain

$$
\frac{d}{d t}\|u\|^{2}=2 \int_{\Gamma}\left(u u_{n}+n_{2} u u_{x_{1}}\right) d s-2 \int_{\Omega}\left(u_{x_{1}} u_{x_{1}}+u_{x_{1}} u_{x_{2}}+u_{x_{2}} u_{x_{2}}\right) d x .
$$

We must show that

$$
\int_{\Gamma} n_{1} u u_{x_{2}} d s=\int_{\Gamma} n_{2} u u_{x_{1}} d s
$$

in order for the energy method to be well defined. Using the definitions above gives

$$
\begin{aligned}
& \int_{\Gamma} n_{1} u u_{x_{2}} d s=\int_{\Gamma}\left(n_{1} n_{2} u u_{n}+n_{1} \tau_{2} u u_{\tau}\right) d s, \\
& \int_{\Gamma} n_{2} u u_{x_{1}} d s=\int_{\Gamma}\left(n_{1} n_{2} u u_{n}+n_{2} \tau_{1} u u_{\tau}\right) d s .
\end{aligned}
$$

Clearly, (1.20) will follow if and only if

$$
\int_{\Gamma} n_{1} \tau_{2} u u_{\tau} d s=\int_{\Gamma} n_{2} \tau_{1} u u_{\tau} d s
$$


From (1.19) and $n_{1}^{2}+n_{2}^{2}=1$ it follows immediately that

$$
\int_{\Gamma} n_{1} \tau_{2} u u_{\tau} d s=\int_{\Gamma} n_{2} \tau_{1} u u_{\tau} d s-\int_{\Gamma} u u_{\tau} d s
$$

Note that the second integral of the right-hand side would vanish identically if $\Gamma$ were smooth. To simplify the analysis, it will, as usual, be assumed that $u$ is supported only in a neighborhood of the lower left corner. Hence, it will be sufficient to consider the boundary portions $\Gamma_{1}$ and $\Gamma_{4}$ corresponding to $\xi_{2}=0$ and $\xi_{1}=0$. Parameterizing $\Gamma$ in the positive direction gives (cf. (1.3))

$$
\int_{\Gamma} u u_{\tau} d s=\frac{1}{2} \int_{1}^{0}-\left(u^{2}\right)_{\xi_{2}} d \xi_{2}+\frac{1}{2} \int_{0}^{1}\left(u^{2}\right)_{\xi_{1}} d \xi_{1}
$$

Letting $\xi_{2} \rightarrow-\xi_{2}$ in the first integral of the right member gives $\left(\partial / \partial \xi_{2} \rightarrow\right.$ $\left.-\partial / \partial \xi_{2}, d \xi_{2} \rightarrow d \xi_{2}\right)$

$$
\int_{\Gamma} u u_{\tau} d s=\frac{1}{2} \int_{-1}^{0}\left(u^{2}\right)_{\xi_{2}} d \xi_{2}+\frac{1}{2} \int_{0}^{1}\left(u^{2}\right)_{\xi_{1}} d \xi_{1}=0
$$

and (1.20) follows. The energy method is thus well defined, and we have

$$
\frac{d}{d t}\|u\|^{2} \leq 2 \int_{\Gamma}\left(\left(1+n_{1} n_{2}\right) u u_{n}+n_{1}^{2} u u_{\tau}\right) d s-\left(\left\|u_{x_{1}}\right\|^{2}+\left\|u_{x_{2}}\right\|^{2}\right) .
$$

The quantity $n_{1}^{2}$ is discontinuous at the corners. Define the jump discontinuity

$$
\left[n_{1}^{2}\right](x)=n_{1 R}^{2}(x)-n_{1 L}^{2}(x),
$$

where $n_{1 R}^{2}(x)$ and $n_{1 L}^{2}(x)$ are the left and right limits of $n_{1}^{2}$ at $x$ (according to the positive orientation of $\Gamma$ ). Straightforward computations show that

$$
\int_{\Gamma} n_{1}^{2} u u_{\tau} d s=\frac{1}{2} \sum_{i=1}^{4}\left[n_{1}^{2}\right]\left(x_{c_{i}}\right) u^{2}\left(x_{c i}, t\right)-\frac{1}{2} \int_{\Gamma}\left(n_{1}^{2}\right)_{\tau} u^{2} d s
$$

where $x_{c i}, i=1, \ldots, 4$, are the corner points. Thus,

$$
\begin{aligned}
\frac{d}{d t}\|u\|^{2} \leq & \sum_{i=1}^{4}\left[n_{1}^{2}\right]\left(x_{c_{i}}\right) u^{2}+\int_{\Gamma}\left(2\left(1+n_{1} n_{2}\right) u u_{n}-\left(n_{1}^{2}\right)_{\tau} u^{2}\right) d s \\
& -\left(\left\|u_{x_{1}}\right\|^{2}+\left\|u_{x_{2}}\right\|^{2}\right) .
\end{aligned}
$$

From this inequality it is obvious that giving Dirichlet data at the corners and Neumann data at the remaining boundaries would yield an energy estimate. In fact, we could even allow inhomogeneous Dirichlet data at the corners and still obtain an energy estimate in terms of the data. The effect of the corners disappears if and only if $\left[n_{1}^{2}\right](x)=0$, which happens if and only if

(i) $n_{1 L}(x)=n_{1 R}(x)$,

(ii) $n_{1 L}(x)=-n_{1 R}(x)$.

The first case implies that the normal is continuous, i. e., $x$ is not a corner point. The second case is more interesting, since the normal is discontinuous, but the effect on the energy estimate disappears. This illustrates how the geometry can interact with the cross terms. The simplest example is obtained by solving 
(1.18) on $\Omega$ being the square with $(1,0),(0,1),(-1,0)$, and $(0,-1)$ as vertices. Evidently, the second case holds at the corners, and no corner values should appear in the energy estimate. This can also be seen by a change of coordinates:

$$
\begin{aligned}
& \xi_{1}=\frac{1}{\sqrt{2}} x_{1}+\frac{1}{\sqrt{2}} x_{2}, \\
& \xi_{2}=-\frac{1}{\sqrt{2}} x_{1}+\frac{1}{\sqrt{2}} x_{2} .
\end{aligned}
$$

Equation (1.18) is then transformed into

$$
u_{t}=\frac{3}{2} u_{\xi_{1} \xi_{1}}+\frac{1}{2} u_{\xi_{2} \xi_{2}}, \quad \xi \in\left(-\frac{1}{\sqrt{2}}, \frac{1}{\sqrt{2}}\right) \times\left(-\frac{1}{\sqrt{2}}, \frac{1}{\sqrt{2}}\right) .
$$

The cross term has vanished; instead the equation has become anisotropic. In this coordinate system it is apparent that no tangential derivatives - and consequently no point values - will appear when deriving the energy estimate.

To solve (1.18) by means of finite difference methods, it is rewritten in selfadjoint form:

$$
\left(J^{-1} u\right)_{t}=\sum_{k=1}^{2}\left(J^{-1}\left(1+n_{1}^{(k)} n_{2}^{(k)}\right) \partial \xi_{k} \cdot \partial u\right)_{\xi_{k}}+\sum_{k \neq l}^{2}(-1)^{l}\left(n_{1}^{(k)} n_{1}^{(k)} u_{\xi_{l}}\right)_{\xi_{k}},
$$

where $n^{(k)}=-\partial \xi_{k} /\left|\partial \xi_{k}\right|$. This equation is discretized in space the usua! way. The cross terms

$$
(-1)^{l}\left(n_{1}^{(k)} n_{1}^{(k)} u_{\xi_{l}}\right)_{\xi_{k}}
$$

must be integrated twice to eliminate the tangential derivatives. In the semidiscrete case this amounts to performing summation by parts twice, the second of which will require the introduction of a commutator, thereby obliterating strict stability (except for the second-order accurate difference operator). To restore strict stability, it would be tempting to reformulate the critical terms in skew-symmetric form:

$$
n_{1}^{(k)} n_{1}^{(k)} u_{\xi_{l}}=\frac{1}{2}\left(n_{1}^{(k)} n_{1}^{(k)} u\right)_{\xi_{l}}+\frac{1}{2} n_{1}^{(k)} n_{1}^{(k)} u_{\xi_{l}}-\frac{1}{2}\left(n_{1}^{(k)} n_{1}^{(k)}\right)_{\xi_{l}} u .
$$

Doing so, however, would introduce lower-order energy terms $\langle\cdot, \cdot\rangle_{h}$, whose presence would destroy strict stability. The simplest way to resolve this ambiguity is to assume homogeneous Dirichlet data, in which case the boundary terms vanish identically, and (1.21) would be the preferred choice. The choice of homogeneous Dirichlet data to eliminate the influence of the mixed derivatives arises naturally when solving the Navier-Stokes equations, since at solid boundaries we have zero velocity, and since the cross terms involve only the velocity components. 
We now turn to general parabolic systems subject to homogeneous Dirichlet conditions,

$$
\begin{array}{ll}
u_{t}=\sum_{i, j=1}^{2} A_{i j} u_{x_{i} x_{j}}+F, & x \in \Omega, \\
u(x, 0)=f(x), & x \in \Gamma . \\
u(x, t)=0, &
\end{array}
$$

This equation can be rewritten in curvilinear coordinates as

$$
\begin{array}{ll}
\left(J^{-1} u\right)_{t}=\sum_{i, j, k=1}^{2}\left(J^{-1} \frac{\partial \xi_{k}}{\partial x_{i}} A_{i j} u_{x_{j}}\right)_{\xi_{k}}-\sum_{j=1}^{2} J^{-1} \operatorname{div}\left(A_{j}\right) u_{x_{j}}+J^{-1} F, & x \in \Omega, \\
u(x, 0)=f(x), & x \in \Gamma, \\
u(x, t)=0, & x \in
\end{array}
$$

where $\operatorname{div}\left(A_{j}\right)=\left(A_{1 j}\right)_{x_{1}}+\left(A_{2 j}\right)_{x_{2}}$. Define $C_{j} \equiv \operatorname{div}\left(A_{j}\right)$. The semidiscrete system is then given by

$$
\left(J^{-1} v\right)_{t}=P\left(\sum_{i, j, k=1}^{2} D_{k} J^{-1} M_{i k} A_{i j} \tilde{D}_{j} v-\sum_{j=1}^{2} J^{-1} C_{j} \tilde{D}_{j} v+J^{-1} F\right) .
$$

The projection operator $P$ represents the homogeneous Dirichlet conditions.

Proposition 1.3. The approximation (1.23) is strictly stable.

Proof. Left to the reader.

\section{INHOMOGENEOUS BOUNDARY CONDITIONS}

The principle for handling inhomogeneous boundary data is best illustrated by means of a simple example. Consider the one-dimensional advection equation

$$
\begin{aligned}
& u_{t}+u_{x}=0, \quad x \in(0,1), \\
& u(x, 0)=f(x), \\
& u(0, t)=g(t) .
\end{aligned}
$$

The corresponding semidiscrete system reads

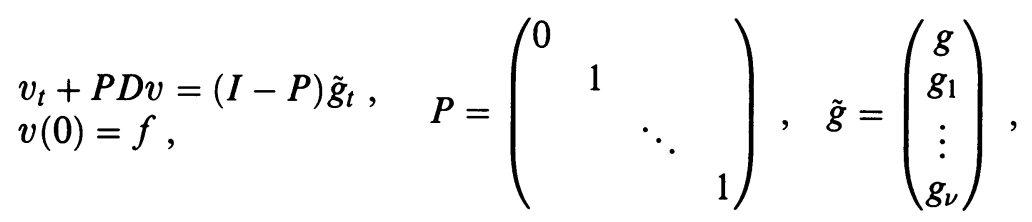

where $g_{j}, j=1, \ldots, \nu$, are to be determined later. Obviously, $v_{0}(t)=g(t)$ if $f_{0}=g(0)$. The boundary condition is thus fulfilled at all time. More generally, multiplying equation (2.2) by $P$ (using $P^{2}=P$ ) and subtracting the resulting equation from (2.2), we can see that $v=P v+(I-P) \tilde{g} \Longleftrightarrow(I-P)(v-\tilde{g})=0$. 
Hence, the energy method gives

$$
\frac{d}{d t}\|v\|_{h}^{2}=-2(v-(I-P) \tilde{g}, D v)_{h}+2\left(v,(I-P) \tilde{g}_{t}\right)_{h} .
$$

Subtracting $2\left(\tilde{g}, v_{t}\right)_{h}$ from both sides, we get

$$
\begin{aligned}
2\left(v-\tilde{g}, v_{t}\right)_{h}= & -2(v-\tilde{g}, D v)_{h}-2\left(\tilde{g}, v_{t}+P D v-(I-P) \tilde{g}_{t}\right)_{h} \\
& +2\left(v-\tilde{g},(I-P) \tilde{g}_{t}\right)_{h} .
\end{aligned}
$$

Using (2.2) and $(I-P)(v-\tilde{g})=0$ shows that

$$
2\left(v-\tilde{g}, v_{t}\right)_{h}=-2(v-\tilde{g}, D v)_{h},
$$

i. e.,

$$
2\left(v-\tilde{g},(v-\tilde{g})_{t}\right)_{h}=-2(v-\tilde{g}, D(v-\tilde{g}))_{h}-2\left(v-\tilde{g}, \tilde{g}_{t}+D \tilde{g}\right)_{h} .
$$

If $\tilde{g}$ solves the auxiliary problem

$$
\begin{aligned}
& \tilde{g}_{t}+D \tilde{g}=0, \\
& \tilde{g}(0)=f,
\end{aligned}
$$

then

$$
\frac{d}{d t}\|v-\tilde{g}\|_{h}^{2}=-2(v-\tilde{g}, D(v-\tilde{g}))_{h}=\left(v_{0}-g\right)^{2}-\left(v_{\nu}-g_{\nu}\right)^{2} \leq 0
$$

since $v_{0}=g$. Thus,

$$
\|v(t)-\tilde{g}(t)\|_{h} \leq\|v(0)-\tilde{g}(0)\|_{h}=0 .
$$

Consequently,

$$
v(t)=\tilde{g}(t), \quad t \geq 0 .
$$

If $\tilde{g}$ satisfies (2.3), we get the energy estimate

$$
\frac{d}{d t}\|\tilde{g}\|_{h}^{2}=-2(\tilde{g}, D \tilde{g})_{h}=g^{2}-g_{\nu}^{2}
$$

Hence,

$$
\|\tilde{g}(t)\|_{h}^{2}+\int_{0}^{t} g_{\nu}^{2}(\tau) d \tau=\|f\|_{h}^{2}+\int_{0}^{t} g^{2}(\tau) d \tau .
$$

Finally, $v=\tilde{g}$ implies

$$
\|v(t)\|_{h}^{2}+\int_{0}^{t} v_{\nu}^{2}(\tau) d \tau=\|f\|_{h}^{2}+\int_{0}^{t} g^{2}(\tau) d \tau
$$

which is identical to the continuous estimate.

It remains to be verified under what circumstances $\tilde{g}$ solves the auxiliary problem (2.3). Let $\hat{g}=\left(\begin{array}{llll}g_{0} & g_{1} & \ldots & g_{\nu}\end{array}\right)^{T}$ be the solution to (2.3). Hence,

$$
D^{j} \hat{g}(0)=D^{j} f, \quad j=0,1, \ldots,
$$

and

$$
\frac{\partial^{j} \hat{g}}{\partial t^{j}}+(-1)^{j+1} D^{j} \hat{g}=0, \quad t \geq 0, \quad j=0,1, \ldots
$$


i. e., for $t=0$ we get the compatibility conditions

$$
\frac{\partial^{j} \hat{g}}{\partial t^{j}}(0)+(-1)^{j+1} D^{j} f=0, \quad j=0,1, \ldots .
$$

Thus, if we require that the initial-boundary data $f$ and $g$ satisfy

$$
\frac{\partial^{j} g}{\partial t^{j}}(0)+(-1)^{j+1}\left(D^{j} f\right)_{0}=0, \quad j=0,1, \ldots,
$$

it follows that

$$
\frac{\partial^{j} g}{\partial t^{j}}(0)=\frac{\partial^{j} g_{0}}{\partial t^{j}}(0), \quad j=0,1, \ldots
$$

By virtue of being the solution to $(2.3), g_{0}(t)$ is analytic in $t$. Hence, if the boundary data $g(t)$ is taken to be analytic, these equalities imply that $g(t)=$ $g_{0}(t), t \geq 0$, i.e., $\tilde{g}=\hat{g}$, which proves that $\tilde{g}$ indeed solves (2.3).

In what follows we shall analyze the general case. Consider the ODE system

$$
\begin{aligned}
& \left(J^{-1} v\right)_{t}=P R(t, v)+(I-P)\left(J^{-1} \tilde{g}\right)_{t}, \\
& v(0)=f,
\end{aligned}
$$

with $J^{-1}$ being the inverse Jacobian, and where

$$
R(t, v)=G(t, v)+J^{-1} F(t), \quad G(t, 0)=0 .
$$

This form arises naturally when discretizing a nonlinear PDE in space; $\tilde{g}$ represents the boundary data, and $F$ is the forcing function; $G(t, v)$ is the discretization of the differential operator. It should be pointed out that most operators $G$ occurring in practice are autonomous, i. e., $G=G(v)$. We use the tilde notation to emphasize that $\tilde{g}$ is only partially determined, that is, some components are determined by the boundary data of the underlying PDE; the remaining components are unknown. It is no restriction to assume that $\tilde{g}=\left(\begin{array}{llll}g_{0} & \ldots & g_{\nu}\end{array}\right)^{T}$ with $g_{i}, i=0, \ldots, s$, being the known components; $s$ is of course independent of the meshsize. Otherwise, $\tilde{g}$ could be brought to this form by permuting the dependent variables appropriately. As usual, we require that $P$ and $J^{-1}$ commute, which is true if the grid is locally isochoric at the boundary. Next, we define the auxiliary problem

$$
\begin{aligned}
& \left(J^{-1} w\right)_{t}=R(t, w), \\
& w(0)=f .
\end{aligned}
$$

Any solution to $(2.5)$ will satisfy

$$
\frac{d^{j}}{d t^{j}}\left(J^{-1} w\right)=R_{j}(t, w), \quad j=0,1, \ldots,
$$

where $R_{j}$ is defined recursively by

$$
\begin{aligned}
& R_{j}(t, w)=\frac{\partial R_{j-1}}{\partial t}(t, w)+\frac{\partial R_{j-1}}{\partial w}(t, w) J R(t, w), \quad j=1,2, \ldots, \\
& R_{0}(t, w)=J^{-1} w .
\end{aligned}
$$

Consequently, at $t=0$ we have

$$
\frac{d^{j}}{d t^{j}}\left(J^{-1} w\right)(0)=R_{j}(0, f), \quad j=0,1, \ldots
$$


Assumption 2.1. The boundary data $g_{i}(t), i=0, \ldots, s$, the initial data $f$, and the forcing function $F$ satisfy the compatibility conditions

$$
\frac{d^{j}}{d t^{j}}\left(J^{-1} \tilde{g}\right)_{i}(0)=\left(R_{j}(0, f)\right)_{i}, \quad i=0, \ldots, s, \quad j=0,1, \ldots .
$$

If $R$ is sufficiently well behaved, in particular if $G$ is linear and autonomous, then $w(t)$ will be analytic for $0 \leq t<T$. Thus, if we require that the boundary data $g_{i}(t), i=0, \ldots, s$, be analytic, it follows that

$$
g_{i}(t)=w_{i}(t), \quad i=0, \ldots, s .
$$

Furthermore, the unknown components $g_{i}, i>s$, are of course taken to be

$$
g_{i}(t)=w_{i}(t), \quad i>s .
$$

Hence, $\tilde{g}=w$ solves (2.5).

Remark. It suffices to consider $g_{i}$ piecewise analytic, since the process can be repeated at $t=t_{1}$, where $t_{1}$ is the critical time when analyticity is lost.

Proposition 2.1. Let $v$ be the solution to (2.4) and suppose that Assumption 2.1 holds. If the boundary data $g_{i}(t), i=0, \ldots, s$, are piecewise analytic in $t$, then

$$
\left\langle v-\tilde{g},(v-\tilde{g})_{t}\right\rangle_{h}=(v-\tilde{g}, G(t, v)-G(t, \tilde{g}))_{h} .
$$

Proof. Using (2.4) and $P v=v-(I-P) \tilde{g}$, which is true since $P$ and $J^{-1}$ commute, we readily conclude that

$$
\left\langle v, v_{t}\right\rangle_{h}=(v-\tilde{g}, R(t, v))_{h}+(\tilde{g}, P R(t, v))_{h}+\left(v,(I-P)\left(J^{-1} \tilde{g}\right)_{t}\right)_{h} .
$$

Hence,

$$
\begin{aligned}
\left\langle v-\tilde{g}, v_{t}\right\rangle_{h}= & (v-\tilde{g}, R(t, v))_{h}+\left(\tilde{g},-\left(J^{-1} v\right)_{t}+P R(t, v)\right)_{h} \\
& +\left(v,(I-P)\left(J^{-1} \tilde{g}\right)_{t}\right)_{h},
\end{aligned}
$$

or, using (2.4),

$$
\left\langle v-\tilde{g}, v_{t}\right\rangle_{h}=(v-\tilde{g}, R(t, v))_{h}+\left(v-\tilde{g},(I-P)\left(J^{-1} \tilde{g}\right)_{t}\right)_{h} .
$$

But

$$
\left(v-\tilde{g},(I-P)\left(J^{-1} \tilde{g}\right)_{t}\right)_{h}=\left((I-P)(v-\tilde{g}),\left(J^{-1} \tilde{g}\right)_{t}\right)_{h}=0,
$$

and so

$$
\left\langle v-\tilde{g}, v_{t}\right\rangle_{h}=(v-\tilde{g}, R(t, v))_{h},
$$

which in turn is equivalent to

$\left\langle v-\tilde{g},(v-\tilde{g})_{t}\right\rangle_{h}=(v-\tilde{g}, R(t, v)-R(t, \tilde{g}))_{h}-\left(v-\tilde{g},\left(J^{-1} \tilde{g}\right)_{t}-R(t, \tilde{g})\right)_{h}$.

The assumptions on $\tilde{g}$ imply that $\left(J^{-1} \tilde{g}\right)_{t}=R(t, \tilde{g})$, which proves the proposition. 
2.1. One-dimensional parabolic systems. We consider the parabolic system (lower-order terms are omitted for convenience)

$$
\begin{aligned}
& u_{t}=\left(A u_{x}\right)_{x}+F \\
& u(x, 0)=f \\
& L_{0} u(0, t)+L_{1} u_{x}(0, t)=g(t)
\end{aligned}
$$

where

$$
L_{0}=\left(\begin{array}{c}
L_{0}^{I} \\
L_{0}^{I I}
\end{array}\right), \quad L_{1}=\left(\begin{array}{c}
L_{1}^{I} \\
0
\end{array}\right), \quad g(t)=\left(\begin{array}{c}
g^{I}(t) \\
0
\end{array}\right),
$$

and $u \in \mathrm{R}^{d}, \operatorname{rank}\left(L_{1}^{I}\right)=d_{1}, \operatorname{rank}\left(L_{0}^{I I}\right)=d_{2}, d_{1}+d_{2}=d$. Equation (2.6) is assumed to be strongly parabolic, i. e., there exists a constant $\delta>0$ such that $u^{T} A(x, t) u \geq 2 \delta|u|^{2}$. Furthermore, $A(x, t)$ is assumed to satisfy the following compatibility condition [1, Lemma 7. 2. 1, p. 215]: For $a, b \in \mathrm{R}^{d}$ satisfying $L_{0}^{I I} a=0, L_{1}^{I} b=0$, one has $a^{T} A(0, t) b=0$. The boundary data $g(t)$ is assumed to be piecewise analytic in $t$.

Let the boundary projection $P$ be given by (1.8); the discretized analytic boundary conditions are defined by $L^{T} v \equiv L_{0} v_{0}+L_{1}(D v)_{0}=g(t)$. The hypotheses for $L_{0}, L_{1}$ and the compatibility condition on $A$ imply that $L$ has full rank if the mesh size $h$ is small enough [2, §3. 2]. Hence, there exists a vector $\tilde{g}(t) \in \mathrm{R}^{(\nu+1) d}$ such that $g(t)=L^{T} \tilde{g}(t)$, i. e., $L^{T} \cdot v=g \Longleftrightarrow L^{T}(v-\tilde{g})=0$. The semidiscrete approximation of (2.6) can then be formulated as

$$
\begin{aligned}
& v_{t}=P(D A D v+F)+(I-P) \tilde{g}_{t}, \\
& v(0)=f .
\end{aligned}
$$

Assuming that initial data satisfy $L^{T} f=g$, we have that (multiply (2.7) by $P$ and subtract the result from $(2.7))(I-P)(v-\tilde{g})=0$ or, equivalently, $L^{T} v=L^{T} \tilde{g}$. Thus,

$$
L_{0} v_{0}+L_{1}(D v)_{0}=g
$$

which shows that the analytic boundary conditions are satisfied to some order of accuracy.

Proposition 2.2. If, in addition to the previous hypotheses, Assumption 2.1 holds, then (2.7) is strictly stable.

Proof. We know that $\tilde{g}$ solves the auxiliary problem

$$
\begin{aligned}
& \tilde{g}_{t}=G(t, \tilde{g})+F(t), \\
& \tilde{g}(0)=f,
\end{aligned}
$$

where $G(t, \tilde{g})=D A D \tilde{g}$. Proposition 2.1 then yields (using $J=I$ )

$$
\begin{aligned}
\left(v-\tilde{g},(v-\tilde{g})_{t}\right)_{h} & =(v-\tilde{g}, D A D(v-\tilde{g}))_{h} \\
& \leq-\left(v_{0}-g_{0}\right)^{T} A(D(v-\tilde{g}))_{0}-2 \delta\|D(v-\tilde{g})\|_{h}^{2} .
\end{aligned}
$$

Since $L^{T}(v-\tilde{g})=0$, it follows that

$$
L_{0}^{I I}\left(v_{0}-g_{0}\right)=0 \text {. }
$$

Furthermore, decompose $v_{j}-g_{j}=\left(v_{j}^{\prime}-g_{j}^{\prime}\right)+\left(v_{j}^{\prime \prime}-g_{j}^{\prime \prime}\right)$, where $v_{j}^{\prime}, g_{j}^{\prime} \in$ $\operatorname{ker}\left(L_{1}^{I}\right), v_{j}^{\prime \prime}, g_{j}^{\prime \prime} \in \operatorname{ker}\left(L_{1}^{I}\right)^{\perp}$. According to the compatibility condition on $A$ 
we then obtain

$$
\left(v-\tilde{g},(v-\tilde{g})_{t}\right)_{h} \leq-\left(v_{0}-g_{0}\right)^{T} A\left(D\left(v^{\prime \prime}-\tilde{g}^{\prime \prime}\right)\right)_{0}-2 \delta\|D(v-\tilde{g})\|_{h}^{2} .
$$

Arguing exactly as in the proof of the homogeneous case [2, proof of Proposition 3. 2] gives

$$
\left(D\left(v^{\prime \prime}-\tilde{g}^{\prime \prime}\right)\right)_{0}=-\tilde{L}_{1}^{-1} L_{0}\left(v_{0}-g_{0}\right),
$$

where

$$
\tilde{L}_{1}=\left(\begin{array}{c}
L_{1}^{I} \\
s_{1}^{T} \\
\vdots \\
s_{d_{2}}^{T}
\end{array}\right)
$$

$\left\{s_{j}\right\}$ is a basis in $\operatorname{ker} L_{1}^{I}$, whence $\tilde{L}_{1}$ is invertible. Consequently,

$$
\left(v-\tilde{g},(v-\tilde{g})_{t}\right)_{h} \leq \gamma\left|v_{0}-g_{0}\right|^{2}-2 \delta\|D(v-\tilde{g})\|_{h}^{2} .
$$

By means of the discrete Sobolev inequality we thus arrive at

$$
\left(v-\tilde{g},(v-\tilde{g})_{t}\right)_{h} \leq\left(\frac{\alpha}{2}+\mathscr{O}(h)\right)\|v-\tilde{g}\|_{h}^{2} .
$$

Thus,

$$
\|v(t)-\tilde{g}(t)\|_{h} \leq e^{(\alpha+\mathscr{O}(h)) t}\|v(0)-\tilde{g}(0)\|_{h}=0,
$$

which is equivalent to $v(t)=\tilde{g}(t), t \geq 0$.

To get the final estimate, we consider the auxiliary problem. One obtains

$$
\frac{d}{d t}\|\tilde{g}\|_{h}^{2} \leq-2 g_{0}^{T} A\left(D \tilde{g}^{\prime \prime}\right)_{0}-4 \delta\|D \tilde{g}\|_{h}^{2}+\|\tilde{g}\|_{h}^{2}+\|F\|_{h}^{2}
$$

Now

and so

$$
L_{0} g_{0}+L_{1}\left(D \tilde{g}^{\prime \prime}\right)_{0}=g
$$

Thus,

$$
\left(D \tilde{g}^{\prime \prime}\right)_{0}=-\tilde{L}_{1}^{-1} L_{0} g_{0}+\tilde{L}_{1}^{-1} g
$$

$$
-2 g_{0}^{T} A\left(D \tilde{g}^{\prime \prime}\right)_{0}=2 g_{0}^{T} A \tilde{L}_{1}^{-1} L_{0} g_{0}-2 g_{0}^{T} A \tilde{L}_{1}^{-1} g \leq \gamma\left|g_{0}\right|^{2}+|g|^{2},
$$

where the algebraic inequality $2 x y \leq \epsilon x^{2}+\epsilon^{-1} y^{2}$ was used. This leads to

$$
\frac{d}{d t}\|\tilde{g}\|_{h}^{2}+\left|g_{0}\right|^{2} \leq(\gamma+1)\left|g_{0}\right|^{2}-4 \delta\|D \tilde{g}\|_{h}^{2}+\|\tilde{g}\|_{h}^{2}+|g|^{2}+\|F\|_{h}^{2} .
$$

The coefficients of this estimate are exactly the same as those of the corresponding analytic inequality. Using $\tilde{g}=v$ and eliminating the boundary terms of the right member by means of the Sobolev inequality yields

$$
\frac{d}{d t}\|v\|_{h}^{2}+\left|v_{0}\right|^{2} \leq(\alpha+\mathscr{O}(h))\|v\|_{h}^{2}+|g|^{2}+\|F\|_{h}^{2}
$$

where $\alpha$ is the same constant as in the analytic estimate. Finally, integration with respect to time results in

$$
\|v\|_{h}^{2}+\int_{0}^{t}\left|v_{0}(\tau)\right|^{2} d \tau \leq e^{(\alpha+\mathcal{O}(h)) t}\left(\|f\|_{h}^{2}+\int_{0}^{t}\left(|g(\tau)|^{2}+\|F(\tau)\|_{h}^{2}\right) d \tau\right),
$$

which is the desired estimate. 
Remark. The boundary conditions are used twice - first in conjunction with Proposition 2.1 to show that $\tilde{g}=v$, and second with the auxiliary problem to get the actual estimate. It should be emphasized that the hypotheses preceding the formulation of Proposition 2.2 are needed in order to prove an energy estimate for the analytic problem (2.6). We do not claim that the additional assumption in the previous proposition is necessary.

2.2. Two-dimensional symmetric hyperbolic systems. We consider equation (1.12) with the lower-order terms omitted for convenience. The boundary conditions are given by $L(x) u(x, t)=g(x, t), x \in \Gamma$, where the analytic boundary operator $L(x)$ is identical to that of $\S 1.1$. The discrete boundary operator is defined as

$$
\begin{aligned}
\left(L^{T} v\right)_{i j}=g\left(x_{i j}, t\right), \quad i=0, \nu, \quad j & =1, \ldots, \nu-1, \quad \text { and } \\
j & =0, \nu, \quad i=0, \ldots, \nu,
\end{aligned}
$$

or, in global form,

$$
L^{T} v=g .
$$

Since $L$ has full rank, it follows that there exists a vector $\tilde{g}(t)$ such that $L^{T} \tilde{g}=$ $g$. Thus,

$$
L^{T}(v-\tilde{g})=0 .
$$

Equation (1.12) is discretized as

$$
\begin{aligned}
& \left(J^{-1} v\right)_{t}=P\left(\frac{1}{2} \sum_{i=1}^{2}\left(D_{i} J^{-1} B_{i} v+J^{-1} B_{i} D_{i} v\right)+J^{-1} F\right)+(I-P)\left(J^{-1} \tilde{g}\right)_{t} \\
& v(0)=f,
\end{aligned}
$$

where $P$ is the orthogonal projection corresponding to the global operator $L^{T}$.

Proposition 2.3. Suppose that Assumption 2.1 holds. Then (2.8) is strictly stable.

Proof. By Assumption 2.1, $\tilde{g}$ solves the auxiliary problem

$$
\left(J^{-1} \tilde{g}\right)_{t}=G(t, \tilde{g})+J^{-1} F(t)
$$

where

$$
G(t, \tilde{g})=\frac{1}{2} \sum_{i=1}^{2}\left(D_{i} J^{-1} B_{i} \tilde{g}+J^{-1} B_{i} D_{i} \tilde{g}\right) .
$$

Hence, according to Proposition 2.1 we have

$$
\left\langle v-\tilde{g},(v-\tilde{g})_{t}\right\rangle_{h}=\frac{1}{2} \sum_{i=1}^{2}\left(v-\tilde{g},\left(D_{i} J^{-1} B_{i}+J^{-1} B_{i} D_{i}\right)(v-\tilde{g})\right)_{h} .
$$

Summation by parts yields

$$
\left\langle v-\tilde{g},(v-\tilde{g})_{t}\right\rangle_{h}=\frac{1}{2}\left\langle v-\tilde{g},\left(n_{1} A_{1}+n_{2} A_{2}\right)(v-\tilde{g})\right\rangle_{\Gamma} .
$$


But $L^{T}(v-\tilde{g})=0$, whence $v-\tilde{g}$ satisfies the homogeneous boundary conditions. Consequently (cf. the proof of Proposition 1.1),

$$
\left\langle v-\tilde{g},\left(n_{1} A_{1}+n_{2} A_{2}\right)(v-\tilde{g})\right\rangle_{\Gamma} \leq-\gamma|v-\tilde{g}|_{\Gamma}^{2} \leq 0 .
$$

Thus, $\tilde{g}(t)=v(t), t \geq 0$.

In the second part of the proof we apply the energy method to the auxiliary problem. Straightforward computations show that

$$
\frac{d}{d t}\langle\tilde{g}, \tilde{g}\rangle_{h}=\left\langle\tilde{g},\left(n_{1} A_{1}+n_{2} A_{2}\right) \tilde{g}\right\rangle_{\Gamma}+2\langle\tilde{g}, F\rangle_{h} .
$$

Take an arbitrary point $x_{0 j}$ on the boundary portion where $\xi_{1}=0$. We must analyze the quadratic form

$$
\tilde{g}_{0 j}^{T}\left(n_{1}^{(1)} A_{1}+n_{2}^{(1)} A_{2}\right)_{0 j} \tilde{g}_{0 j} .
$$

We know that $L^{T} \tilde{g}=g$, where $g$ is the vector representing the analytic boundary data. Define $\varphi_{i j}=Q^{T}\left(x_{i j}\right) \tilde{g}_{i j}$. Hence,

$$
\left(\varphi_{i j}\right)_{I}=S\left(x_{i j}\right)\left(\varphi_{i j}\right)_{I I}+g_{i j},
$$

and the quadratic form is transformed into

$$
\tilde{g}_{0 j}^{T}\left(n_{1}^{(1)} A_{1}+n_{2}^{(1)} A_{2}\right)_{0 j} \tilde{g}_{0 j}=\varphi_{0 j}^{T} \Lambda_{0 j} \varphi_{0 j} .
$$

Using (2.9) gives (omitting the spatial subscripts for simplicity)

$$
\varphi^{T} \Lambda \varphi=\varphi_{I I}^{T}\left(\Lambda_{I I}+S^{T} \Lambda_{I} S\right) \varphi_{I I}+2 \varphi_{I I}^{T} S^{T} \Lambda_{I} g+g^{T} \Lambda_{I} g .
$$

It is assumed that $\Lambda_{I I} \leq-\gamma$ at $x_{0 j}$. For sufficiently small $S$ we thus get

$$
\varphi^{T} \Lambda \varphi \leq-\frac{\gamma}{2}\left|\varphi_{I I}\right|^{2}+\left(1+\left|\Lambda_{I}\right|\right)|g|^{2} .
$$

Now, $\left|\varphi_{I}\right| \leq|S|\left|\varphi_{I I}\right|+|g|$. Hence,

$$
\varphi^{T} \Lambda \varphi+\frac{\gamma}{4}|\varphi|^{2} \leq-\frac{\gamma}{4}\left|\varphi_{I I}\right|^{2}+\frac{\gamma}{4}\left|\varphi_{I}\right|^{2}+\left(1+\left|\Lambda_{I}\right|\right)|g|^{2} \leq\left(3+\left|\Lambda_{I}\right|\right)|g|^{2}
$$

for $S$ small enough. It should be underscored that this is exactly the same estimate one gets in the continuous case. At each boundary point $x_{0 j}$ we have thus established that

$$
\tilde{g}_{0 j}^{T}\left(n_{1}^{(1)} A_{1}+n_{2}^{(1)} A_{2}\right)_{0 j} \tilde{g}_{0 j}+\frac{\gamma_{0 j}}{4}\left|\tilde{g}_{0 j}\right|^{2} \leq\left(3+\left|\left(\Lambda_{0 j}\right)_{I}\right|\right)\left|g_{0 j}\right|^{2}
$$

with a similar expression at points $x_{i 0}$ corresponding to $\xi_{2}=0$. Letting $\inf \left(\gamma_{i j}\right) \equiv \gamma>0$, we thus obtain

$$
\frac{d}{d t}\langle\tilde{g}, \tilde{g}\rangle_{h}+\frac{\gamma}{4}\langle\tilde{g}, \tilde{g}\rangle_{\Gamma} \leq\left(3+|\Lambda|_{\infty}\right)\langle g, g\rangle_{\Gamma}+2\langle\tilde{g}, F\rangle_{h} .
$$


Finally, with the identification $v=\tilde{g}$, integration gives the energy estimate

$$
\begin{aligned}
\langle v(t), & v(t)\rangle_{h}+\int_{0}^{t}\langle v(\tau), v(\tau)\rangle_{\Gamma} d \tau \\
& \leq K e^{t}\left(\langle f, f\rangle_{h}+\int_{0}^{t}\left(\langle g(\tau), g(\tau)\rangle_{\Gamma}+\langle F(\tau), F(\tau)\rangle_{h}\right) d \tau\right),
\end{aligned}
$$

which proves the theorem.

Remark. Because of the terms $\varphi_{I I}^{T} S^{T} \Lambda_{I} g+g^{T} \Lambda_{I} g$, the constant $K$ of the energy estimate will in general satisfy $K>1$, even if no estimate of the boundary terms $\langle v, v\rangle_{\Gamma}$ is wanted. For $g=0$, i. e., homogeneous boundary conditions, the critical terms disappear, and one may take $K=1$ in case no boundary estimate is needed.

\section{BIBLIOGRAPHY}

1. Heinz-Otto Kreiss and Jens Lorenz, Initial-boundary value problems and the Navier-Stokes equations, Pure and Applied Mathematics, vol. 136, Academic Press, San Diego, CA, 1989.

2. Pelle Olsson, Summation by parts, projections, and stability. I, Math. Comp. 64 (1995), 10351065.

RiaCS, Mail Stop T20G-5, NASA Ames Research Center, Moffett Field, California 94035-1000

E-mail address: pelle@riacs.edu 